\title{
CORRIGENDUM
}

\section{Biophysical controls on organic carbon fluxes in fluvial networks}

Tom J. Battin, Louis A. Kaplan, Stuart Findlay, Charles S. Hopkinson, Eugenia Marti, Aaron I. Packman, J. Denis Newbold and Francesc Sabater

Nature Geosci. 1, 95-100 (2008); published online: 20 January 2008; corrected after print: 20 July 2009.

In the version of this Progress Article originally published, Table 1 was incorrect and should have been as shown here.

Table 1 Gross primary production (GPP), respiration (R) and net ecosystem production (NEP) in streams, rivers and estuaries as determined from whole-ecosystem metabolism measurements (see Supplementary Information S2), and global estimates of respiration and net heterotrophy.

\begin{tabular}{|c|c|c|c|c|c|}
\hline Ecosystem & $\begin{array}{l}\text { GPP } \\
\left(\mathrm{gC} \mathrm{m}^{-2} \mathrm{~d}^{-1}\right)\end{array}$ & 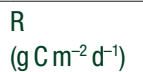 & $\begin{array}{l}\text { NEP } \\
\left(\mathrm{gC} \mathrm{m}^{-2} \mathrm{~d}^{-1}\right)\end{array}$ & $\begin{array}{l}\text { Global R } \\
\left(\mathrm{PgCy}^{-1}\right)\end{array}$ & $\begin{array}{l}\text { Global net heterotrophy } \\
\left(\mathrm{PgCy}^{-1}\right)\end{array}$ \\
\hline $\begin{array}{l}\text { Streams } \\
(n=62)\end{array}$ & $\begin{array}{l}0.73 \pm 0.14^{\star} \\
(0.02-5.62)\end{array}$ & $\begin{array}{l}1.93 \pm 0.19^{\star} \\
(0.29-8.16)\end{array}$ & $\begin{array}{l}-1.20 \pm 0.15^{\star} \\
(-5.86-2.51)\end{array}$ & 0.19 & 0.12 \\
\hline $\begin{array}{l}\text { River } \\
(\mathrm{n}=37)\end{array}$ & $\begin{array}{l}0.91 \pm 0.10^{\dagger} \\
(0.06-2.28)\end{array}$ & $\begin{array}{l}1.53 \pm 0.15^{\star} \\
(0.20-3.54)\end{array}$ & $\begin{array}{l}-0.66 \pm 0.11^{\dagger} \\
(-2.06-1.60)\end{array}$ & 0.16 & 0.07 \\
\hline $\begin{array}{l}\text { Estuaries } \\
(\mathrm{n}=31)\end{array}$ & $\begin{array}{l}3.14 \pm 0.41^{\ddagger} \\
(0.72-10.4)\end{array}$ & $\begin{array}{l}3.51 \pm 0.32^{\dagger} \\
(0.83-7.58)\end{array}$ & $\begin{array}{l}-0.39 \pm 0.21^{\dagger} \\
(-2.98-2.86)\end{array}$ & 1.20 & 0.13 \\
\hline
\end{tabular}

Subsequently, the values of global fluvial respiration and global net heterotrophy reported in the text on page 97 were incorrect.

These errors have been corrected in the HTML and PDF versions. 